\title{
31. PULSAR DISTANCES, SPIRAL STRUCTURE AND THE INTERSTELLAR MEDIUM
}

\author{
B. Y. MILLS \\ Cornell-Sydney University Astronomy Center, University of Sydney, Australia
}

\begin{abstract}
The distances of all pulsars are calculated on the assumption that they are immersed in a uniform medium of average electron density $0.06 \mathrm{~cm}^{-3}$. It then appears that the pulsars are concentrated towards the local and Sagittarius spiral features and that their mean height above the plane is consistent with that of known supernova remnants. The mean distances appear to be approximately correct, but individual distances are uncertain by about a factor of two. Evidence from radio continuum results supports this model of the ionized interstellar medium.
\end{abstract}

Several attempts have been made to estimate the distances of the pulsars using theoretically based models for the free electron distribution in the interstellar medium. A different approach is to make a crude first order approximation that the pulsars are immersed in a uniform dispersing medium, calculate the distances and directions of all pulsars on this assumption and compare the results with the properties of other galactic components, particularly supernova remnants, which would appear to have a common origin. Discrepancies revealed in this comparison may then be used to refine the assumed model of the interstellar medium and improve the distance scale.

For reasons discussed later I have taken the free electron density of the uniform medium to be $n_{\mathrm{e}}=0.06 \mathrm{~cm}^{-3}$. In Figure 1 the distribution of the 40 pulsars of known dispersion measure has been plotted as projected on to the galactic plane, on the assumption that their distances are directly proportional to their dispersion measures $\left(\int n_{\mathrm{e}} \mathrm{d} l\right)$, using the value of $n_{\mathrm{e}}$ above. The pulsar parameters are given in Table I.

If the pulsars and the dispersing medium are uniformly distributed throughout the galactic disc, it would be expected that the distribution in Figure 1 would show circular symmetry. It does not. We may disregard the absence of high dispersion pulsars in the smaller sector between $l^{\mathrm{II}}=55^{\circ}$ and $l^{\mathrm{II}}=195^{\circ}$ because this area is outside the field of view of the Molonglo Radio Telescope. This instrument is responsible for the discovery of all but one of the high dispersion pulsars in the remaining sector. However, in this larger sector the distribution also does not appear symmetric and displays features which appear to be closely related to the well known Hi spiral pattern.

Practically all the pulsars may be associated with the two groupings shown on the diagram which reproduce quite well in direction and distance the local and Sagittarius spiral features. The high dispersion pulsars marking extensions of these features appear to be particularly significant. This grouping appears too striking to be ascribed to chance, and the crude first order approximation to the free electron distribution would seem to be more accurate than one might reasonably expect.

With this suggestion in mind let us look briefly at some of the relevant observed properties of the interstellar medium. Firstly, it has been shown by Gould (1969) that the free-free emission and absorption processes in the ionized medium agree with 
TABLE I

The galactic coordinates and derived distances of 40 pulsars

\begin{tabular}{|c|c|c|c|c|c|}
\hline Designation & $\begin{array}{l}l \\
\mathrm{o}\end{array}$ & $\begin{array}{l}b \\
o\end{array}$ & $\int_{\mathrm{cm}^{-3} \mathrm{pc}} n_{\mathrm{e}} \mathrm{d} l$ & $\begin{array}{l}z^{\mathrm{a}} \\
\mathrm{pc}\end{array}$ & $\begin{array}{l}d^{\mathrm{a}} \\
\mathrm{pc}\end{array}$ \\
\hline $\begin{array}{l}\text { MP } 0031 \\
\text { MP } 0254 \\
\text { CP } 0328 \\
\text { MP } 0450 \\
\text { NP } 0527\end{array}$ & $\begin{array}{l}111 \\
271 \\
145 \\
217 \\
184\end{array}$ & $\begin{array}{r}-69 \\
-55 \\
0 \\
-34 \\
-\quad 7\end{array}$ & $\begin{array}{l}12 \\
10 \\
27 \\
25 \\
50\end{array}$ & $\begin{array}{r}-186 \\
-137 \\
0 \\
-253 \\
-102\end{array}$ & $\begin{array}{r}70 \\
96 \\
450 \\
346 \\
830\end{array}$ \\
\hline $\begin{array}{l}\text { NP } 0532 \\
\text { PSR } 0628-28 \\
\text { MP } 0736 \\
\text { CP } 0808 \\
\text { AP } 0823+26\end{array}$ & $\begin{array}{l}185 \\
237.0 \\
254 \\
140 \\
197\end{array}$ & $\begin{array}{l}-6 \\
-16.7 \\
-9 \\
+34 \\
+32\end{array}$ & $\begin{array}{r}56 \\
10 \\
100 \\
6 \\
19\end{array}$ & $\begin{array}{l}-98 \\
-48 \\
-260 \\
+\quad 56 \\
+168\end{array}$ & $\begin{array}{r}930 \\
16 C \\
1640 \\
83 \\
268\end{array}$ \\
\hline $\begin{array}{l}\text { PSR } 0833-45 \\
\text { CP } 0834 \\
\text { MP } 0835 \\
\text { MP } 0940\end{array}$ & $\begin{array}{l}264 \\
219.7 \\
260 \\
278\end{array}$ & $\begin{array}{c}-3 \\
+26.3 \\
0 \\
-\quad 3\end{array}$ & $\begin{array}{r}63 \\
13 \\
120 \\
145\end{array}$ & $\begin{array}{r}-54 \\
+\quad 95 \\
0 \\
-127\end{array}$ & $\begin{array}{r}1050 \\
194 \\
2000 \\
2420\end{array}$ \\
\hline $\begin{array}{ll}\text { PP } & 0943 \\
\text { CP } & 0950 \\
\text { MP } & 0959 \\
\text { CP } & 1133 \\
\text { MP } & 1154\end{array}$ & $\begin{array}{l}230 \\
228.9 \\
281 \\
241.9 \\
297\end{array}$ & $\begin{array}{l}+45 \\
+43.7 \\
-1 \\
+69.2 \\
-0.1\end{array}$ & $\begin{array}{c}9.5 \\
3 \\
90 \\
5 \\
270\end{array}$ & $\begin{array}{l}+112 \\
+\quad 34 \\
-\quad 26 \\
+\quad 78 \\
-\quad 8\end{array}$ & $\begin{array}{r}112 \\
34 \\
1500 \\
30 \\
4500\end{array}$ \\
\hline $\begin{array}{l}\text { AP } 1237+25 \\
\text { MP } 1240 \\
\text { MP } 1426 \\
\text { MP } 1449 \\
\text { PSR } 1451-68\end{array}$ & $\begin{array}{l}250 \\
302.0 \\
313 \\
315.3 \\
313.9\end{array}$ & $\begin{array}{l}+86 \\
-1.0 \\
-6 \\
-5.3 \\
-8.6\end{array}$ & $\begin{array}{c}8.5 \\
220 \\
60 \\
90 \\
12\end{array}$ & $\begin{array}{l}+142 \\
-64 \\
-104 \\
-138 \\
-\quad 23\end{array}$ & $\begin{array}{r}10 \\
3660 \\
1000 \\
1500 \\
198\end{array}$ \\
\hline $\begin{array}{l}\text { HP } 1507 \\
\text { MP } 1530 \\
\text { AP } 1541+9 \\
\text { MP } 1642\end{array}$ & $\begin{array}{r}90 \\
326 \\
18 \\
15\end{array}$ & $\begin{array}{l}+53 \\
+\quad 2 \\
+46 \\
+26\end{array}$ & $\begin{array}{l}15.5 \\
20 \\
35 \\
40\end{array}$ & $\begin{array}{l}+206 \\
+\quad 12 \\
+420 \\
+282\end{array}$ & $\begin{array}{l}234 \\
332 \\
405 \\
600\end{array}$ \\
\hline $\begin{array}{l}\text { MP } 1706 \\
\text { MP } 1727 \\
\text { MP } 1747 \\
\text { PSR } 1749-28 \\
\text { MP } 1818\end{array}$ & $\begin{array}{c}7 \\
341 \\
344 \\
1.6 \\
25\end{array}$ & $\begin{array}{l}+15 \\
-9 \\
-11 \\
-1.0 \\
+5\end{array}$ & $\begin{array}{r}10 \\
140 \\
40 \\
51 \\
70\end{array}$ & $\begin{array}{l}+43 \\
-365 \\
-128 \\
-15 \\
+103\end{array}$ & $\begin{array}{r}161 \\
2420 \\
655 \\
850 \\
1160\end{array}$ \\
\hline $\begin{array}{l}\text { MP } 1911 \\
\text { CP } 1919 \\
\text { PSR } 1929+10 \\
\text { JP } 1933+16 \\
\text { AP } 2015+28\end{array}$ & $\begin{array}{l}31 \\
55.8 \\
48 \\
52 \\
68\end{array}$ & $\begin{array}{l}+7 \\
+3.5 \\
-4 \\
-2 \\
-4\end{array}$ & $\begin{array}{c}75 \\
12.6 \\
8 \\
143 \\
14\end{array}$ & $\begin{array}{l}+152 \\
+\quad 13 \\
-\quad 9 \\
-\quad 87 \\
-\quad 16\end{array}$ & $\begin{array}{r}1240 \\
210 \\
134 \\
2500 \\
233\end{array}$ \\
\hline $\begin{array}{l}\text { PSR } 2045-16 \\
\text { PSR } 2218+47\end{array}$ & $\begin{array}{l}30.5 \\
98\end{array}$ & $\begin{array}{l}-33.1 \\
-8\end{array}$ & $\begin{array}{l}11 \\
44\end{array}$ & $\begin{array}{l}-105 \\
-102\end{array}$ & $\begin{array}{l}162 \\
726\end{array}$ \\
\hline
\end{tabular}

a In the final columns are listed $z=\frac{\int n_{\mathrm{e}} \mathrm{d} l}{0.06} \sin b$; and $d=\frac{\int n_{\mathrm{e}} \mathrm{d} l}{0.06} \cos b$ 
radio observations of emission at high radio frequencies and absorption at low frequencies if an electron temperature of about $6000 \mathrm{~K}$ is assumed. There is no evidence for a significant component of low temperature electrons produced by cosmic ray ionization, except possibly in some directions of high $\mathrm{HI}$ concentration.

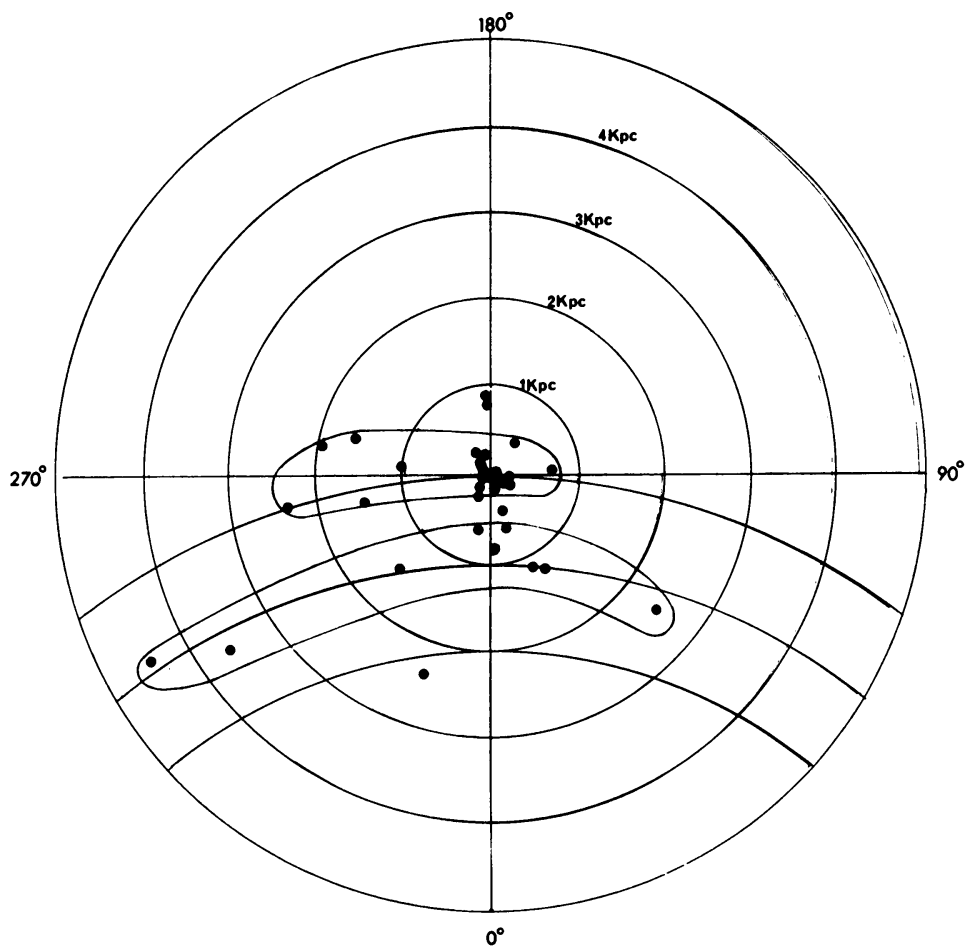

Fig. 1. The distribution of pulsars in the galactic plane on the assumption of a uniform dispersing medium of density $\left\langle n_{\mathrm{e}}\right\rangle=0.06 \mathrm{~cm}^{-3}$.

Gould also finds that the local value of the mean square electron density $\left\langle n_{\mathrm{e}}^{2}\right\rangle \simeq$ $0.06 \mathrm{~cm}^{-6}$. However, we have seen that a plausible distribution of pulsars is yielded by $\left\langle n_{\mathrm{e}}\right\rangle=0.06 \mathrm{~cm}^{-3}$ or $\left\langle n_{\mathrm{e}}\right\rangle^{2}=0.0036 \mathrm{~cm}^{-6}$. Distances derived from this value of $n_{\mathrm{e}}$ are also consistent with a common origin for pulsars and supernova remnants. The former have a mean height above the plane, $\langle|z|\rangle \simeq 110 \mathrm{pc}$, while for the supernova remnants in the same part of the Galaxy $\langle|z|\rangle \simeq 85 \mathrm{pc}$ according to a recent tabulation of Milne (1969). In view of the uncertainty in both distance scales the agreement is good and the distributions in $|z|$ otherwise appear very similar. The identifications of the Vela (PSR 0835-45) and Crab (NP 0532) pulsars with the corresponding supernova remnants yield $\left\langle n_{\mathrm{e}}\right\rangle \simeq 0.1$ and $\left\langle n_{\mathrm{e}}\right\rangle \simeq 0.03$ respectively. Accordingly, there appears little doubt that, in the interstellar medium, $\left\langle n_{\mathrm{e}}^{2}\right\rangle \gg\left\langle n_{\mathrm{e}}\right\rangle^{2}$. This inequality implies that the distribution of the ionized medium is very irregular, but we have seen that the dispersion measures appear to give a good indication of pulsar distances. We must suppose that the free-free processes $\left(\propto\left\langle n_{\mathrm{e}}^{2}\right\rangle\right)$ are dominated by dense clouds and the 
pulsar dispersions $\left(\propto\left\langle n_{\mathrm{e}}\right\rangle\right)$ by a much more uniform tenuous intercloud medium. The temperature of the ionized intercloud medium cannot be substantially less than that of the clouds or its effects would be more apparent in the low frequency absorption measurements. Thus ionization by dilute UV radiation seems very likely.

Further evidence for a hot intercloud medium comes from some recent $\mathrm{H}$-line observations of Radhakrishnan and Murray (1969), who find that the intercloud hydrogen atoms are at a very much higher spin temperature than those in the $\mathrm{HI}$ clouds responsible for the greater part of the observed galactic emission and absorption of the $\mathrm{H}$-line. In one direction at least the spin temperature exceeds $1000 \mathrm{~K}$.

A plasma with $\left\langle n_{\mathrm{e}}\right\rangle \simeq 0.06 \mathrm{~cm}^{-3}$ and $T \simeq 6000 \mathrm{~K}$ is unlikely to be closely confined to the galactic plane, certainly not if there is any tendency towards equipartition of turbulent energies. Ultraviolet ionization would be possible to great heights and, in general, one might expect such a plasma to exist at heights at least as great as those to which the magnetic fields extend. A measure of this extent is given by the galactic distribution of synchrotron radiation which is much wider than that of the $\mathrm{H}$-line emission, for which the mean height $\langle|z|\rangle$ is similar to that of the pulsars. Accordingly, the assumption that all pulsars lie within the dispersing layer appears to be very reasonable. If this were not so it would be difficult to explain the resulting very high $z$ distances of some of the pulsars.

A better fit to the Sagittarius arm distance and the Vela and Crab supernovae is obtained if it is assumed that, within the spiral arms, the mean electron density is greater than $0.06 \mathrm{~cm}^{-3}$ and, between the arms, it is less. However, the small amount of available data does not appear to justify the assignment of actual numbers at this stage. Even if improved statistical distances can be obtained there appears little prospect of appreciably reducing the uncertainty in the distances of individual pulsars, indeed the only refinement which appears justifiable is an allowance for the dispersion measure of known Hil regions when they lie in front of a pulsar. Only two of the pulsars, PSR 0833-45 and MP 0736, are obviously affected but the $\mathrm{H}$ II region has low surface brightness and is believed to be very close so that corrections should not be large. Quantitative information is not at present available.

To conclude, it appears that the simplest possible model for the dispersing medium yields distances which are probably individually accurate to a factor of two and yields the pulsar statistics with much greater accuracy. There is a striking association of a group of ten pulsars with the accepted location of the Sagittarius spiral arm. The great majority of the remainder appears to be associated with the local spiral feature. This interpretation of the data follows from the assumption of a fairly uniform distribution of free electrons extending to much greater heights above the plane than the dense Hi clouds.

\section{References}

Gould, R. J.: 1969, Australian J. Phys. 22, 189.

Milne, D. K.: 1969, 'Non-thermal Galactic Radio Sources', submitted to the Australian J. Phys.

Radhakrishnan, V. and Murray, J. D.: 1969, Proc. Astron. Soc. Australia 1, 215. 